\title{
Human eIF4AIII interacts with an eIF4G-like partner, NOM1, revealing an evolutionarily conserved function outside the exon junction complex
}

\author{
Andrei Alexandrov, David Colognori, and Joan A. Steitz ${ }^{1}$ \\ Department of Molecular Biophysics and Biochemistry, Howard Hughes Medical Institute, Yale University School of Medicine, \\ New Haven, Connecticut 06536, USA
}

\begin{abstract}
Despite the lack of an exon junction complex (EJC), Saccharomyces cerevisiae contains Fal1p, a DEAD-box helicase highly homologous to eIF4AIII. We show that yeast Fal1p is functionally orthologous to human eIF4AIII, since expression of human eIF4AIII complements both the lethal phenotype and the 18S rRNA biogenesis defect of $f a l 1 \Delta$ (null) yeast. We further show that yeast Fal1p interacts genetically with an eIF4G-like protein, Sgd1p: One allele of $s g d 1$ acts as a dominant extragenic suppressor of a mutation in a predicted RNA-binding residue of Fal1p, whereas another synthetically exacerbates the growth defect of this fal1 mutation. Both sgd1 mutations map to a single, short, evolutionarily conserved patch that matches key eIF4A-interacting residues of eIF4G when superimposed on the X-ray structure of the eIF4A/eIF4G complex. We demonstrate direct physical interactions between yeast Sgd1p and Fal1p, and between their human orthologs (NOM1 and eIF4AIII) in vitro and in vivo, identifying human NOM1 as a missing eIF4G-like interacting partner of eIF4AIII. Knockdown of eIF4AIII and NOM1 in human cells demonstrates that this novel conserved eIF4A/eIF4G-like complex acts in pre-rRNA processing, adding to the established functions of eIF4A/eIF4G in translation initiation and of eIF4AIII as the core component of the EJC.
\end{abstract}

[Keywords: eIF3AIII; NOM1; FAL1; SGD1; exon junction complex; ribosome biogenesis]

Supplemental material is available for this article.

Received July 20, 2010; revised version accepted March 28, 2011.

RNA helicases are ubiquitously present in organisms ranging from viruses to humans. Helicases participate in numerous aspects of RNA metabolism, including transcription, editing, mRNA splicing, 3 '-end formation, export, mRNA translation initiation and termination, quality control, degradation, and ribosome biogenesis (Rocak and Linder 2004). Known molecular functions of RNA helicases include unwinding of RNA duplexes (Ray et al. 1985), removal of mRNP protein components (Fairman et al. 2004), serving as RNA clamps (Ballut et al. 2005), annealing RNA strands (Yang and Jankowsky 2005), and stabilizing RNA folding intermediates (Solem et al. 2006). For some of these functions, the energy of NTP hydrolysis is strictly required. For others, like serving as RNA clamps or placeholders, ATP hydrolysis appears to be dispensable (Ballut et al. 2005; Shibuya et al. 2006). Despite their variety (Saccharomyces cerevisiae, for example, has 39 putative helicases) and often essential roles, the exact

${ }^{1}$ Corresponding author.

E-MAIL joan.steitz@yale.edu; FAX (203) 624-8213.

Article is online at http://www.genesdev.org/cgi/doi/10.1101/gad.2045411. functions, substrates, and relevant interacting partners of RNA helicases are known in only a few cases (Bleichert and Baserga 2007). The DEAD-box subfamily is the largest group of RNA helicases, displaying considerable sequence homology, including conservation of helicase motifs and the helicase core (Tanner and Linder 2001). Yet, the DEADbox helicases are very specific: They are not able to complement the deletion of another DEAD-box helicase even when overexpressed (Tanner et al. 2003).

The translation initiation factor eIF4A (encoded by eIF4AI and eIF4AII) is one of the best studied DEAD-box helicases. eIF4A is considered a "minimal" DEAD-box helicase because its core eIF4A-like domain lacks N-terminal and C-terminal extensions (Tanner and Linder 2001). eIF4A functions in cap-dependent translation initiation and is required to melt the secondary structure of the 5' untranslated region (UTR) to allow scanning of the mRNA and recognition of the start codon. It is part of the heterotrimeric complex eIF4F (eIF4G, eIF4A, and eIF4E) (Rozen et al. 1990), where eIF4G is the central scaffolding protein that also binds the $\mathrm{m}^{7} \mathrm{Gppp}$ cap-binding protein eIF4E and recruits the $43 \mathrm{~S}$ preinitiation complex /consisting of the 
40S ribosomal subunit and initiation factors eIF1, eIF1A, eIF2, eIF3, eIF5, and Met-tRNA ${ }^{\text {Ini }}$ ) via its eIF3 subunit (Hinnebusch 2006).

The DEAD-box helicase eIF4AIII has striking homology with eIF4A, but is nevertheless functionally distinct: It has no known role in translation initiation, is localized mostly in the nucleus, does not substitute for eIF4A in in vitro ribosome-binding assays, and inhibits translation in vitro (Li et al. 1999). Human eIF4AIII has been reported to bind eIF4G in vitro (Li et al. 1999), but no functional consequences of this association have been described. Instead, eIF4AIII plays a central role in coupling mRNA splicing to downstream events as a core component of the exon junction complex (EJC). This multiprotein complex is deposited on the mRNA during splicing $\sim 25$ nucleotides (Le Hir et al. 2000a) upstream of the exon-exon junction (Le Hir et al. 2000b). The EJC binds mRNA in a sequence-independent manner and with very high affinity; certain components of the EJC stably associate with mRNA both during nuclear export and later in the cytoplasm. The EJC plays a key role in nonsense-mediated mRNA decay (NMD) (Le Hir et al. 2001; Lykke-Andersen et al. 2001), mRNA localization (Palacios et al. 2004), mRNA export from the nucleus (Le Hir et al. 2001), and the enhancing effect of splicing on translation (Nott et al. 2004). The crystal structure of the EJC core proteins complexed with RNA revealed eIF4AIII's two RecA-like domains enclosing an ATP molecule, with Magoh/Y14 and MLN51 stabilizing eIF4AIII in an ATP-bound and RNA-locked state (Andersen et al. 2006; Bono et al. 2006).

Surprisingly, eIF4AIII-like proteins comprise a separate branch of the evolutionary tree (Li et al. 1999; Linder 2003 ), with $>60 \%$ sequence identity over their entire length between humans and yeast (Fig. 1A). At odds with this conservation are reports that EJC components in Drosophila melanogaster and Caenorhabditis elegans are not required for NMD (Gatfield et al. 2003; Palacios et al. 2004; Longman et al. 2007), whereas S. cerevisiae lacks other known EJC components and NMD in yeast does not depend on splicing (Gonzalez et al. 2001). Instead, the $S$. cerevisiae eIF4AIII-like protein Fallp is essential for $18 \mathrm{~S}$ rRNA biogenesis, specifically for 35S pre-rRNA processing at sites A0, A1, and A2 (Kressler et al. 1997).

Here, using a genetic screen for dominant allele-specific extragenic suppressors of the fal1 cold-sensitive (CS) phenotype, we identified an essential S. cerevisiae complex, Fallp/Sgd1p, which is homologous to eIF4A/eIF4G but acts outside of translation initiation. Instead, it is required for $18 \mathrm{~S}$ rRNA biogenesis. Whereas mutation of Fallp affected growth and pre-rRNA processing at sites A0, A1, and A2, a suppressing mutation in Sgd1p alleviated both the growth and pre-rRNA processing defects. We then demonstrated that human eIF4AIII, which is highly homologous to yeast Fallp, is also a functional ortholog of Fallp, since heterologous expression of human eIF4AIII in yeast specifically complemented growth and rRNA biogenesis defects of FAL1 deletion. Consistent with evolutionary conservation of the Fallp/Sgdlp complex, human NOM1, which is homologous to yeast Sgdlp, interacts directly with eIF4AIII in vivo and in vitro, as demonstrated by coprecipitation of endogenous eIF4AIII with Flag-tagged NOM1 from nuclear extracts of human cells and GST pull-downs of heterologously expressed purified proteins. Consistent with conservation of the function of the complex from yeast to humans, knockdown of either eIF4AIII or NOM1 affects pre-rRNA processing at site 1 in human cells, whereas knockdown of eIF4AIII additionally affects pre-rRNA processing at site 2 . Therefore, depending on its interacting partners, human eIF4AIII plays a dual role in two of the central biogenesis pathways in cells: those leading to rRNA and mRNA.

\section{Results}

Human eIF4AIII specifically complements the lethal phenotype and restores $18 S$ rRNA levels

in falls yeast cells

Since $S$. cerevisiae Fallp is highly homologous to metazoan eIF4AIII (Fig. 1A), we used plasmid shuffle (Supplemental Fig. S1A) to ask whether eIF4AIII from humans and $D$. melanogaster can complement the lethal phenotype of yeast Fallp deletion (Fig. 1B). The observed complementation is specific for eIF4AIII, since other yeast and human DEAD-box helicases tested (human eIF4AI, human eIF4AII, and yeast eIF4A [Tif1p]) do not complement (Fig. 1B). Expression analysis of Protein A-tagged proteins demonstrated that the lack of complementation is not due to lower protein expression (Supplemental Fig. S1B), since the levels of noncomplementing proteins (human eIF4AI and eIF4AII and yeast Tif1p) and of eIF4AIII mutants (K88N and E188Q) are higher than or similar to those of human eIF4AIII(WT).

To confirm the observed complementation, we used a promoter shutoff approach (Fig. 1C; Supplemental Fig. S1C). fal1s strains expressing human eIF4AIII from the GAL1 promoter (fal1 $\Delta \mathrm{pP}_{\text {GAL1 }}$ H.eIF4AIII) are viable when grown on galactose, but not on glucose (vector in Fig. 1C). Introduction of an additional plasmid expressing human eIF4AIII as a GST fusion from the $P_{\text {CUP1 }}$ promoter $\left(\mathrm{Cu}^{2+}\right.$ responsive; expression is increased when $\mathrm{Cu}^{2+}$ is present) rescued growth of the fal1s $\mathrm{pP}_{\mathbf{G A L 1}}$ H.eIF4AIII strain on glucose (H.eIF4AIII in Fig. 1C). Rescue depended on $\mathrm{Cu}^{2+}$ concentration in the medium, whereas double mutations K88A, T89P (Walker A box) and D187E, E188L (Walker B box) in human eIF4AIII completely abolished complementation. Notably, eIF4AIII from D. melanogaster also complemented fal1s dependent on $\mathrm{Cu}^{2+}$, but more weakly than the human protein.

To test function, we asked whether human eIF4AIII can rescue the $18 \mathrm{~S}$ rRNA biogenesis defect reported previously for a fal1 temperature-sensitive (TS) strain (Fig. 2A; Kressler et al. 1997). Indeed, expression of human eIF4AIII from a GAL1 promoter on galactose (but not glucose) restored growth of the fal1 $\Delta$ (null) strain in liquid culture (Fig. 2B,C). 18S rRNA levels were restored to near normal (Fig. 2D, top panel), while levels of 20S rRNA, an $18 \mathrm{~S}$ precursor, were partially restored (Fig. 2D, middle panel) and the accumulation of 23S rRNA was partially relieved (Fig. 2D, bottom panel). Together, these results 
Alexandrov et al.

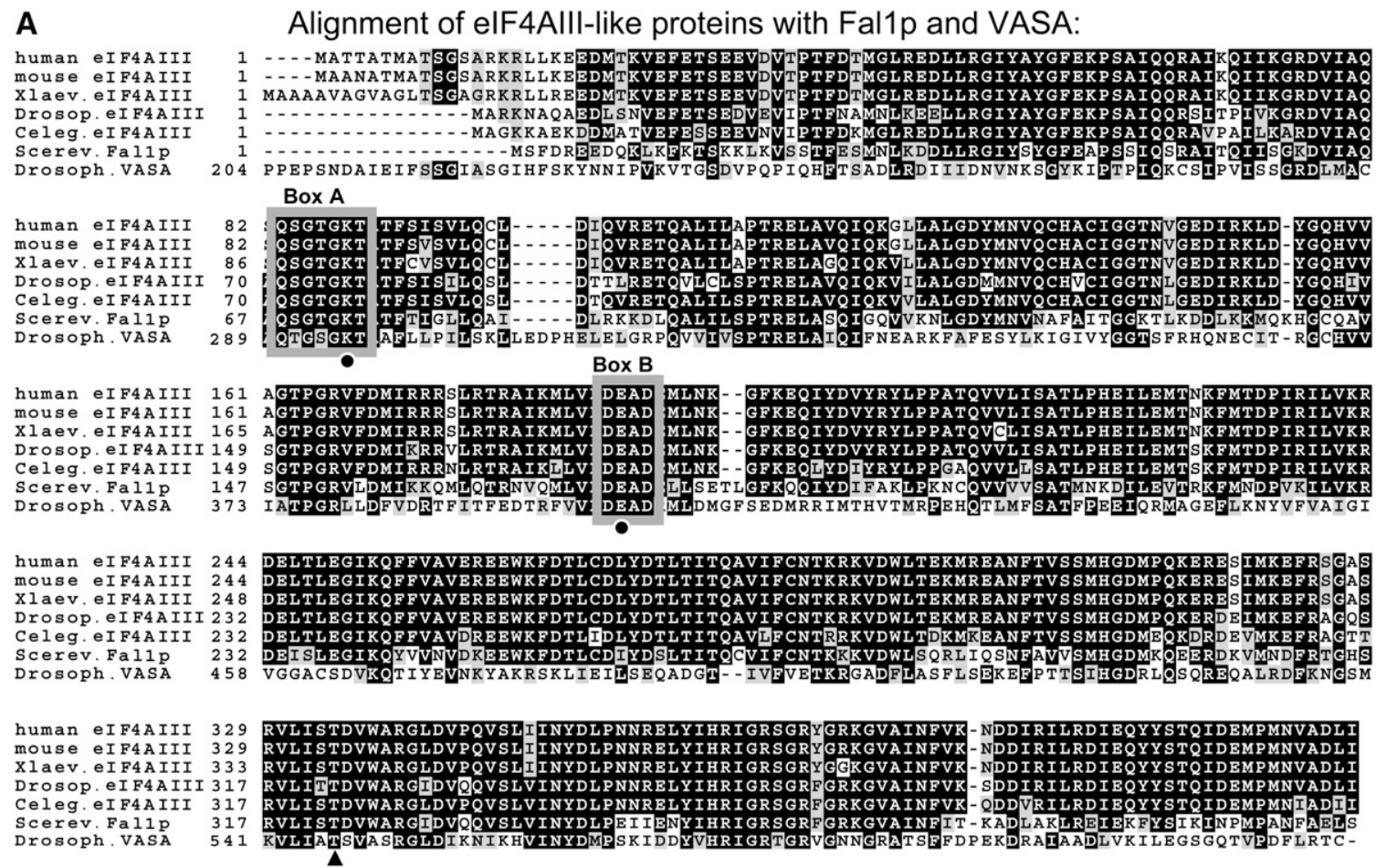

B
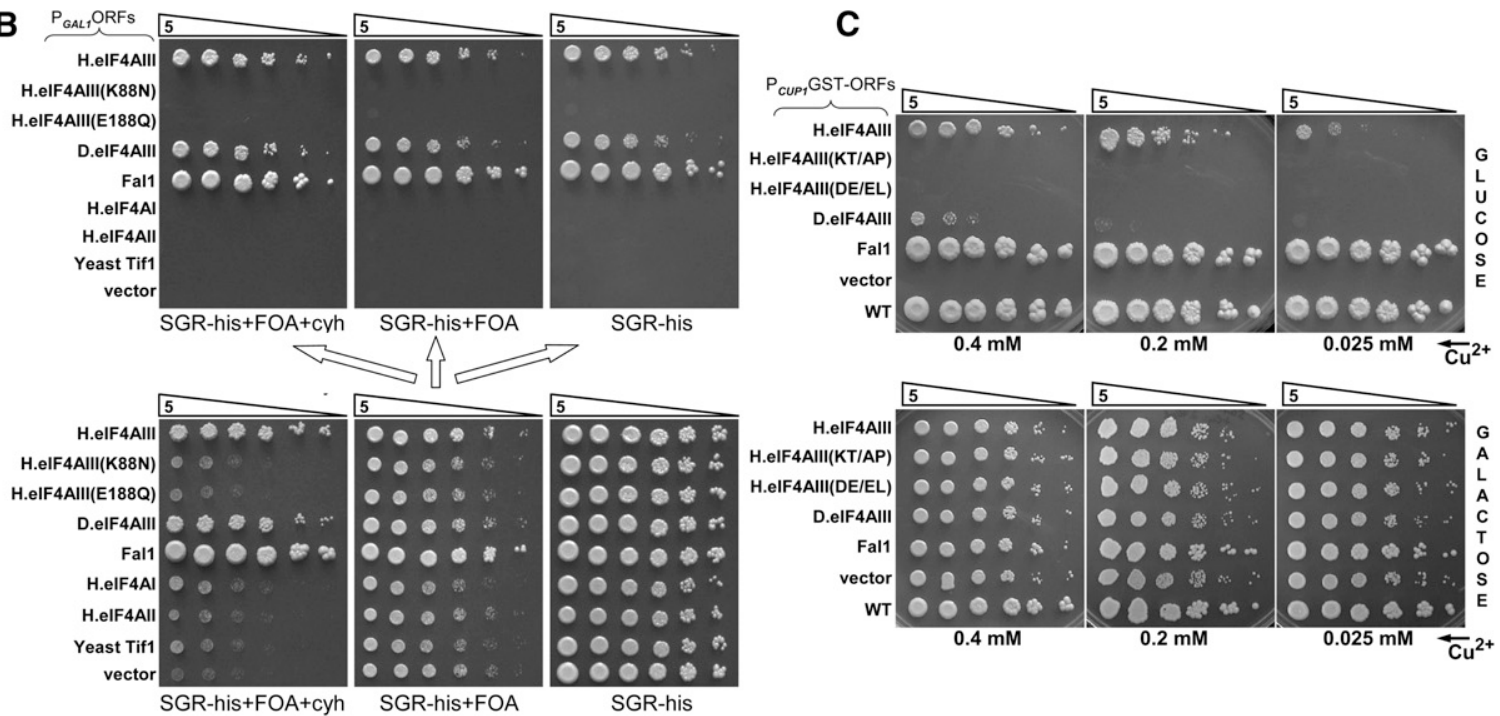

Figure 1. Human eIF4AIII is homologous to and can replace $S$. cerevisiae Fallp in vivo. (A) Amino acid sequence alignment of eIF4AIII homologous proteins from humans, Mus musculus, Xenopus laevis, D. melanogaster, C. elegans, and S. cerevisiae. Walker A and B boxes are shown in gray rectangles. The sequence of D. melanogaster VASA is at the bottom. Also indicated are amino acids in the Walker boxes of human eIF4AIII, in which mutations (K88N and E188Q) were reported previously (Shibuya et al. 2006; Zhang and Krainer 2007) to abolish ATPase activity in vitro, but not NMD in vivo or EJC assembly in vitro (•), and a conserved threonine (T322), whose mutation to valine in $S$. cerevisiae Fallp resulted in a CS phenotype $(\mathbf{A})$. (B) Expression of human or D. melanogaster eIF4AIII complements the lethal phenotype of yeast fal1s, as observed by plasmid shuffle (Supplemental Fig. S1A); Walker A and B boxes are required for complementation. (H.eIF4AIII) human eIF4AIII; [H.eIF4AIII(K88N) and H.eIF4AIII(E188Q)] Walker A and B box mutants of human eIF4AIII; (D.eIF4AIII) D. melanogaster eIF4AIII; (Fall) S. cerevisiae Fallp; (H.eIF4AI and H.eIF4AII) human eIF4AI and eIF4AII. (C) Expression of human and D. melanogaster eIF4AIII complements the lethal phenotype of yeast fal1D, as observed by GAL1 promoter shutoff and activation of expression from a copper-inducible promoter (CUP1) with increasing concentrations of $\mathrm{Cu}^{2+}$ (Supplemental Fig. S1C). (H.eIF4AIII) human eIF4AIII; (KT/AP) K88T, A89P; (DE/EL) D188E, E189L; (D.eIF4AIII) D. melanogaster eIF4AIII; (Fall)S. cerevisiae Fallp. 
A

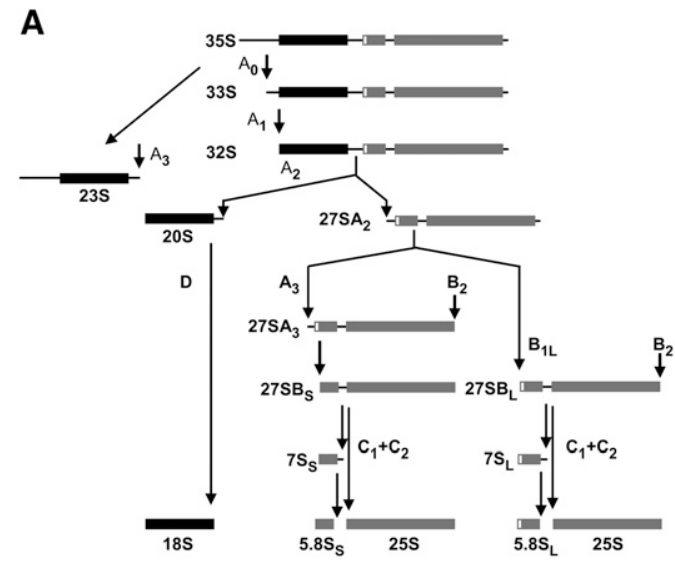

B

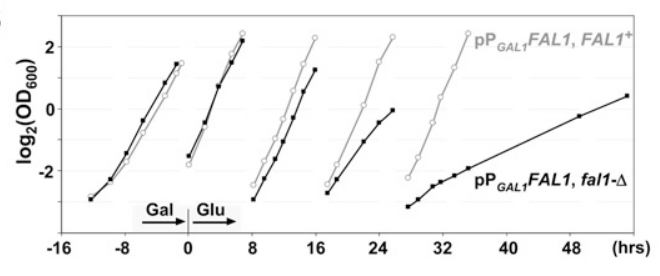

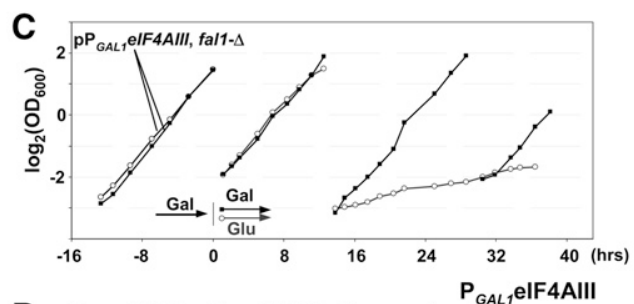

D $\mathrm{P}_{\text {GAL1 }} F A L 1$ P $\mathrm{P}_{\text {GAL1 }}$ FAL1 $\mathrm{P}_{\text {GAL1 }}$ vect. fal1- $\triangle$
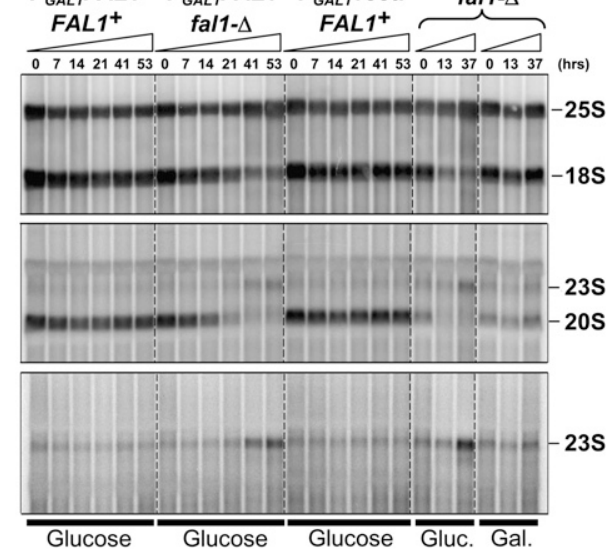

Figure 2. Expression of human eIF4AIII restores $18 \mathrm{~S}$ rRNA biogenesis in the $S$. cerevisiae fal1s strain. (A) Schematic of rRNA processing in $S$. cerevisiae. $(B)$ Depletion of Fallp in yeast $S$. cerevisiae results in a growth defect in liquid culture. $(C)$ Expression of human eIF4AIII from the GAL1 promoter rescues the growth defect of the yeast fal1 $\Delta$ strain in liquid culture. $(D$, top panel) Expression of human eIF4AIII restores 18S rRNA levels in the yeast fal1s strain (probes L2 and L9). (Middle panel) Expression of human eIF4AIII partially restores $20 \mathrm{~S}$ rRNA levels in the yeast fal1 $\Delta$ strain (probe L3). (Bottom panel) Expression of human eIF4AIII partially relieves accumulation of $23 \mathrm{~S}$ rRNA in the yeast fal1s strain (probe L1). Supplemental Figure S2 shows probes.

demonstrate that human eIF4AIII can substitute for Fallp and function in $18 \mathrm{~S}$ ribosome biogenesis in yeast.

Mutations in the conserved Walker $A$ and $B$ boxes

of human eIF4AIII abolish rescue of the falls yeast phenotype

The amino acid sequences of the Walker $A$ and $B$ boxes in human eIF4AIII are completely conserved from yeast to humans (Fig. 1A). Yet, human eIF4AIII bearing K88N and E188Q single mutations in the Walker A and B boxes, respectively, and thus lacking detectable ATPase activity, can surprisingly support NMD in vivo and assemble an EJC in vitro (Shibuya et al. 2006). Moreover, the nonhydrolyzable ATP analog AMP-PNP can substitute for ATP during EJC core assembly in vitro (Ballut et al. 2005; Andersen et al. 2006; Bono et al. 2006), confirming that the ATPase activity of eIF4AIII is not required for these functions and human eIF4AIII bearing the K88A mutation in the Walker A box is active in the Y14/Magoh loading complementation assay despite its lack of helicase activity (Zhang and Krainer 2007).

Since conservation of the Walker A/B boxes among Fallp orthologs is nonetheless striking, we tested the K88N and E188Q mutations in human eIF4AIII for complementation of the yeast fal1s lethal phenotype. Figure $1 \mathrm{~B}$ shows that these mutations completely abolish rescue, highlighting a crucial role of the conserved Walker A and B boxes in a function of human eIF4AIII that is shared with Fallp in this heterologous system. $\operatorname{sgd} 1(S 340 Y)$ is a dominant extragenic suppressor of the T322V mutation in a putative RNA-binding domain of Fal1p

Fallp is considered a "minimal" helicase because it contains only two RecA domains and no extensions (Kressler et al. 1997). This suggests that other factor(s) may be responsible for its substrate specificity. To identify such factor(s) or, possibly, substrates of Fallp, we first attempted a tandem affinity purification (TAP) approach but were not successful, probably because of the transient nature of the interactions. We then focused on genetic screens, aiming to identify spontaneous extragenic dominant allele-specific suppressors of mutations in conserved RNA-interacting amino acids of Fallp (Supplemental Fig. $\mathrm{S} 8 \mathrm{~A}, \mathrm{~B}$ ). An advantage is that interacting partners (suppressors) can be identified if a physical interaction is required for cell survival, regardless of how weak or transient the contact is. Based on the results of our genetic screens (Supplemental Figs. S8-S10), we constructed yeast strains carrying individual chromosomal sgd1(S340Y) and sgd1(N343Y) mutations in the fal1(T322V) background [abbreviated further as fal1(T/V)]. Growth curves (Fig. 3A) and serial dilutions (Fig. 3B; Supplemental Fig. S3A) of these yeast strains demonstrate that $\operatorname{sgd1}(\mathrm{S} 340 \mathrm{Y})$ acts as a dominant (Supplemental Fig. S3A, cf. lanes 3,4 and 5,6) extragenic suppressor of $f a 11(\mathrm{~T} / \mathrm{V})$ CS growth phenotype (Fig. 3B, cf. lanes 2 and 3 at $16^{\circ} \mathrm{C}$ ).

Three lines of evidence demonstrate allele specificity of the suppression of the fal1(T/V) CS phenotype by 
A
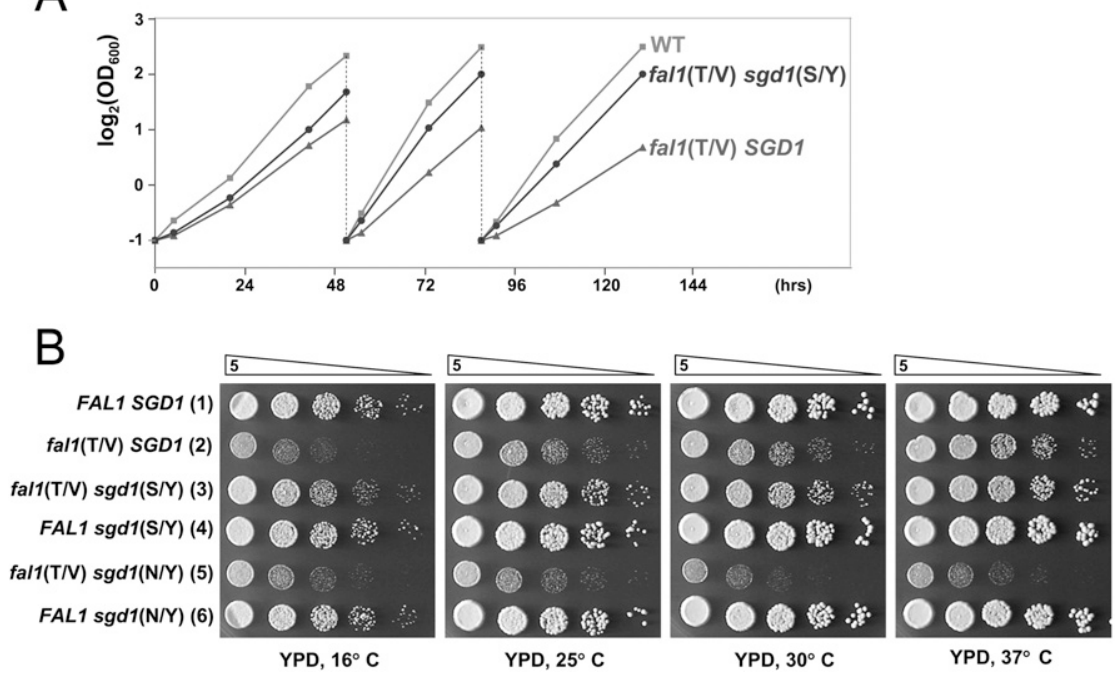

Figure 3. SGD1 interacts genetically with FAL1. (A) Extragenic suppressor sgd1(S340Y) alleviates the growth defect of the CS fal1(T322V) strain in liquid culture (YPD) (strains were exponentially grown at $30^{\circ} \mathrm{C}$ and shifted to $16^{\circ} \mathrm{C}$ at time 0$) .(B)$ Whereas sgd1(S340Y) suppresses the growth defect of the fal1 $(\mathrm{T} 322 \mathrm{~V})$ strain at $16^{\circ} \mathrm{C}$, a nearby mutation $[\operatorname{sgd} 1(\mathrm{~N} 343 \mathrm{Y})]$ in the same protein results in a growth defect synthetic with fal1(T322V) at $37^{\circ} \mathrm{C}(\mathrm{YPD})$.
sgd1(S340Y). First, the phenotype of a different fal1(Q144R, V167T, A194V, M221T, D223G, C272G, A372T, P390T) mutant strain that is TS (Supplemental Fig. S3D,E) was not suppressed at any tested temperature by $\operatorname{sgd1}(\mathrm{S} 340 \mathrm{Y})$, sgd1(N343Y), or SGD1(WT) expressed in multicopy or single copy (Supplemental Fig. S3B,C). Second, neither SGD1(WT), sgd1(S340Y), nor sgd1(N343Y) suppressed the lethal fal1 $\Delta$ (null) phenotype, as demonstrated by plasmid shuffle (Supplemental Fig. S4A,B). Third, whereas the chromosomal sgd1(S340Y) mutation suppressed the fal1(T/V) CS phenotype (Fig. 3B, cf. lanes 2 and 3 at $\left.16^{\circ} \mathrm{C}\right)$, a nearby $\operatorname{sgd} 1(\mathrm{~N} 343 \mathrm{Y})$ mutation had little if any effect on the growth of the fall(T/V) strain at this temperature (Fig. 3B, cf. lanes 2 and 5 at $16^{\circ} \mathrm{C}$ ). Instead, the $\operatorname{sgd1}(\mathrm{N} 343 \mathrm{Y})$ fal1(T/V) double mutant displayed a synthetic growth defect at elevated temperatures (Fig. 3B, cf. lanes 2, 5, and 6 at $37^{\circ} \mathrm{C}$ ); complementation of the synthetic growth defect of the $\operatorname{sgd1}(\mathrm{N} 343 \mathrm{Y})$ fal1(T/V) double mutant by plasmid-expressed $S G D 1$ and FAL1 is shown in Supplemental Figure S4C (cf. lanes 2,5,6 in Fig. 3B and lanes 2-4 in Supplemental Fig. S4C).

Genetically interacting residues in Sgd1p (S340 and N343) identify a conserved interface between yeast Sgd1p/Fal1p and human NOM1/eIF4AIII

The existence of a dominant allele-specific suppressor often reflects a functional physical interaction between two proteins. Sgd1p is highly conserved (Fig. 4A) and is known to be essential in S. cerevisiae (Akhtar et al. 2000), D. melanogaster (http://www.flybase.org), and C. elegans (Maeda et al. 2001); Sgdlp homologs are found in the majority of sequenced eukaryotes (Supplemental Fig. S5), including human. Sgd1p is an eIF4G-like protein (Ponting 2000; Marintchev and Wagner 2005) containing MIF4G and MA3 domains (Fig. 4A). Both MIF4G and MA3 domains in eIF4G were shown previously to bind eIF4A (Morino et al. 2000; Oberer et al. 2005; Schutz et al. 2008). It was therefore plausible that a MIF4G-containing Sgd1p could partner with Fallp, which is $60 \%$ identical to eIF4A (Fig. 1A; Kressler et al. 1997).

Several further lines of evidence suggested a potential physical eIF4G/eIF4A-like interaction between Sgdlp and Fallp in yeast, and between NOM1 and eIF4AIII (their respective orthologs) in humans. First, our dominant allelespecific suppressor mutation, sgd1(ㅈ340Y), and the synthetic mutation $\operatorname{sgd1}(\underline{\mathrm{N}} 343 \mathrm{Y})$ appeared in the MIF4G (the major eIF4A-interacting) domain of Sgd1p (Fig. 4A). Second, within the MIF4G domain itself, these two mutations remarkably appeared two amino acids apart within a 12amino-acid conserved motif (NSSLNKLSDSNI in yeast Sgdlp and KSLLNKLTLEMF in yeast eIF4G [Tiflp]) (Fig. 4A), representing the major surface of eIF4G that contacts eIF4A (Schutz et al. 2008). Together, $\underline{\text { S6}} 612$ and $\underline{N} 615$ of yeast eIF4G (corresponding to S340 and N343 of yeast Sgd1p) form four direct intermolecular hydrogen bonds with eIF4A, based on the X-ray structure of the yeast eIF4A/eIF4G complex (Fig. 4B; Schutz et al. 2008). Third, human NOM1 has been shown previously to interact with eIF4A in yeast two-hybrid screens and pull-down assays (Simmons et al. 2005); an in vitro interaction between GSTtagged NOM1 and eIF4AIII was mentioned as "data not shown" (Simmons et al. 2005).

Here, we present four lines of evidence for a conserved direct eIF4G/eIF4A-like physical interaction between Sgd1p and Fallp in yeast, and between NOM1 and eIF4AIII in humans: First, TAP-Fallp and Myc-Sgdlp specifically coimmunoprecipitate from extracts of formaldehyde cross-linked yeast cells (Fig. 5A; Supplemental Fig. S6A). Second, a comparable specific interaction exists between their human counterparts: Myc-eIF4AIII and Flag-NOM1 coimmunoprecipitate from nuclear extracts of human HEK293 cells (Fig. 5B), but no cross-linking is required. Third, a direct interaction between human eIF4AIII and NOM1 can be reconstituted using heterologously expressed purified proteins (Fig. 5D). Finally, we identified endogenous human eIF4AIII among 24 proteins specifically copurifying from nuclear extract of human HEK293 
A

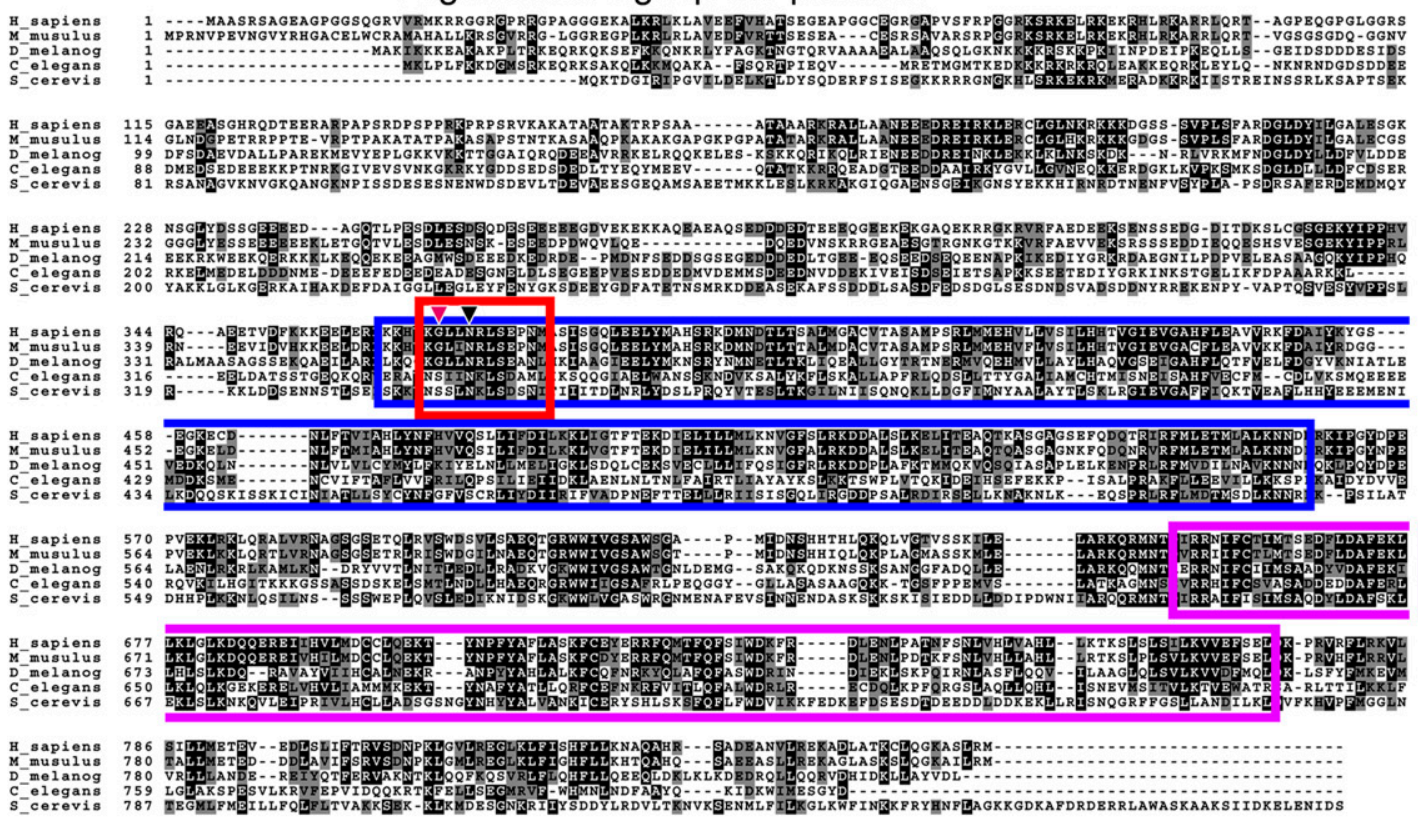

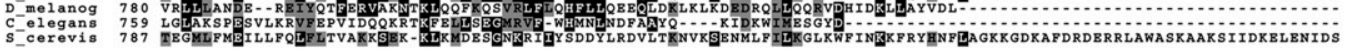

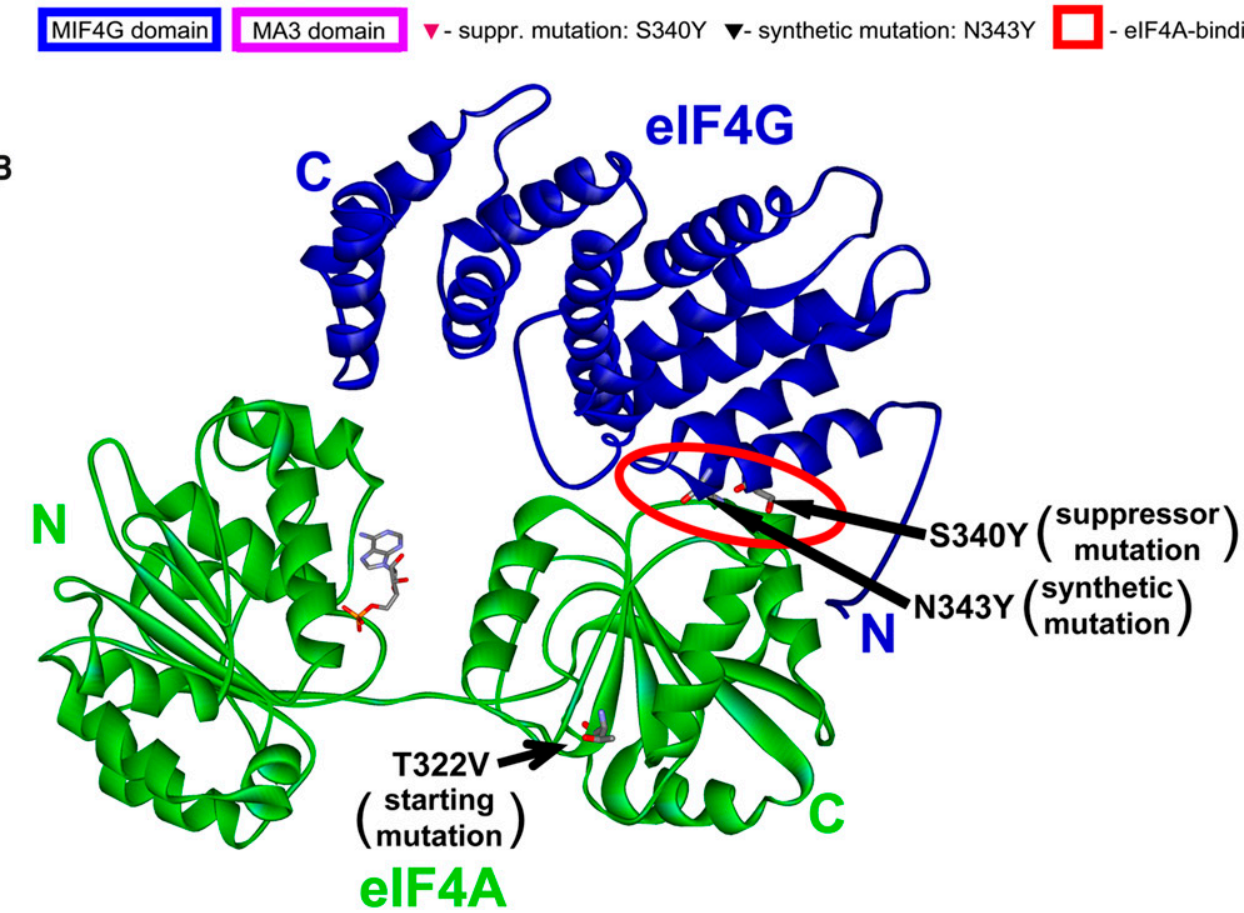

Figure 4. The suppressor mutation $\operatorname{sgd1}(\mathrm{S} 340 \mathrm{Y})$ and synthetic mutation $\operatorname{sgd1}(\mathrm{N} 343 \mathrm{Y})$ map to a known conserved 12-amino-acid motif that comprises the largest interface between eIF4G and eIF4A. (A) Amino acid sequence alignment of $S$. cerevisiae protein Sgd1p with putative Sgd1p-like proteins from humans, M. musculus, D. melanogaster, and C. elegans. Predicted MIF4G and MA3 domains (Ponting 2000) are indicated with blue and purple boxes, respectively. The conserved 12-amino-acid motif (KSLLNKLTLEMF in yeast eIF4G and NSSSLNKLSDSNI in yeast Sgd1p), representing the largest surface of eIF4G interacting with eIF4A (Schutz et al. 2008), is indicated with a red box. Mutations in Sgdlp that result in genetic interactions with fal1(T/V) are indicated with triangles; each of these residues is known to form two intermolecular hydrogen bonds with eIF4A, based on the crystal structure of the eIF4G/eIF4A complex (Schutz et al. 2008). (B) Structure of the eIF4G/eIF4A complex (Schutz et al. 2008) showing the locations of residues homologous to the starting mutation in Fallp (eIF4A) and the suppressor and synthetic mutations in Sgdlp (eIF4G). The major eIF4A/eIF4G interaction interface is marked with a red oval. 

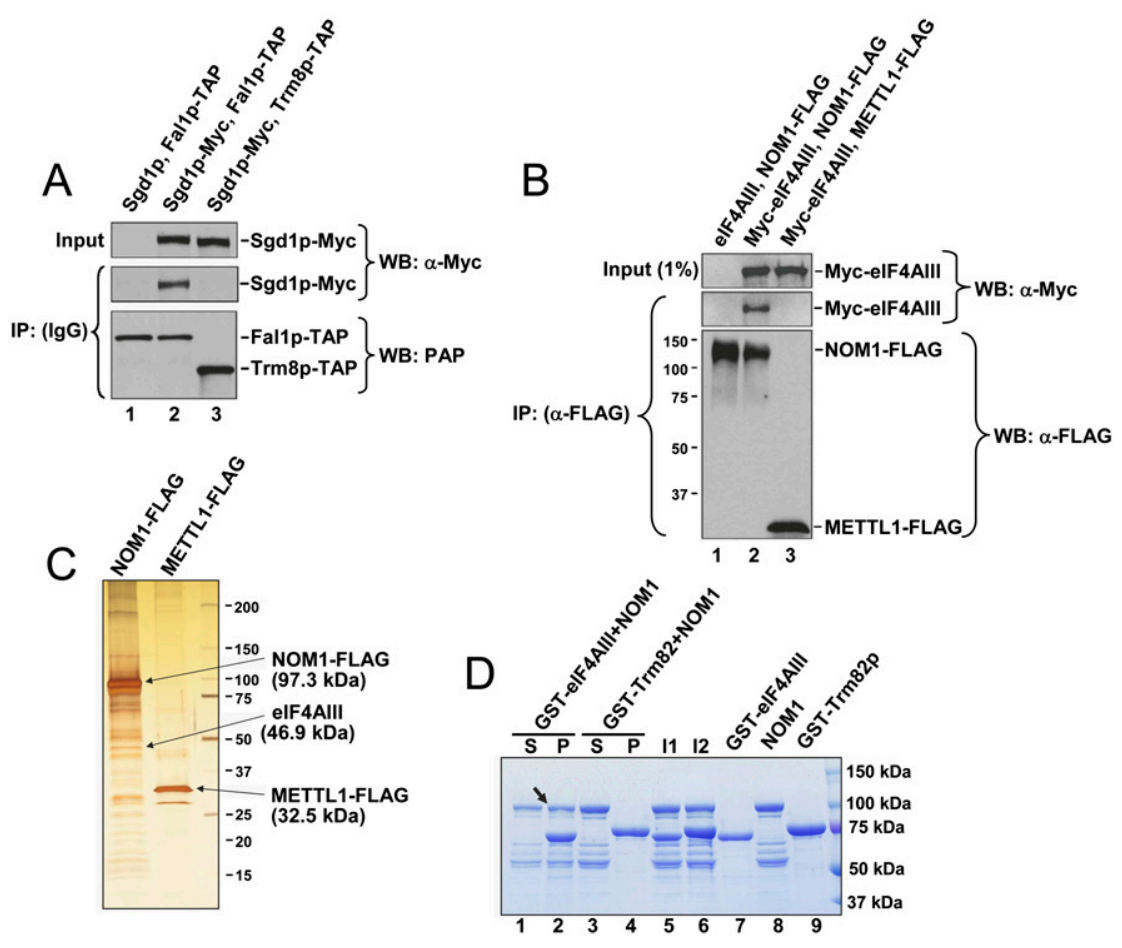

Figure 5. Direct physical interactions between $S$. cerevisiae Fallp and Sgdlp and between human eIF4AIII and NOM1. (A) Yeast Sgd1p and Fallp coimmunoprecipitate. Western blot of IgG precipitates from extracts of formaldehyde cross-linked yeast cells expressing tagged and untagged versions of yeast Sgdlp and Fallp (Supplemental Fig. S6A), as indicated. All proteins were expressed from their own promoters. A TAPtagged yeast protein (Trm8p) with known nuclear localization provided a negative control. TAP-tagged proteins were precipitated using bead-immobilized rabbit IgG (the ZZ domain of Staphylococcus aureus protein A present in the TAP tag binds IgG) (Rigaut et al. 1999). The noncovalent complex of horseradish peroxidase (HRP) with polyclonal rabbit anti-peroxidase (PAP) was used for Western blot detection of TAP-tagged proteins (Puig et al. 2001) (the ZZ domain of protein $\mathrm{A}$ in the TAP tag binds PAP); detection of the c-myc epitope used HRPconjugated mouse monoclonal antibody 9E10. (B) Human eIF4AIII and NOM1 coimmunoprecipitate. Western blot of Flag-purified samples from a nuclear extract of human HEK293 cells expressing transiently transfected tagged and untagged versions of human eIF4AIII and NOM1, as indicated (no cross-linking required). Flag-tagged human protein METTL1, with known nuclear localization provided a negative control. $(C)$ Endogenous human eIF4AIII was identified by mass spectrometry among 24 protein bands specifically copurifying from a nuclear extract of human HEK293 cells with Flag-tagged NOM1 (silver staining) (Supplemental Fig. S6B). ( $D$ ) Direct interaction between heterologously expressed and purified human GST-eIF4AIII and NOM1 is demonstrated by GST pulldown (the gel is Coomassie-stained). The arrow shows the position of full-length NOM1 copurified with GST-eIF4AIII. GST-tagged purified yeast protein Trm82p provided a negative control. (Lane 8) Additional bands in the NOM1 preparation represent $\mathrm{N}$ terminally truncated forms of NOM1. I1 and I2 denote inputs for pull-downs shown in lanes 1 and 2, and lanes 3 and 4, respectively.

cells with Flag-tagged NOM1 (Fig. 5C; Supplemental Fig. S6B) using mass spectrometry.

\section{Sgd1p/Fal1p and NOM1/eIF4AIII form functional complexes acting in $18 S$ rRNA biogenesis \\ in S. cerevisiae and humans, respectively}

Sgd1p and NOM1 are documented nucleolar proteins in both yeast and humans (Akhtar et al. 2000; Simmons et al. 2005); a function for Sgd1p in yeast rRNA biogenesis has been reported ( $\mathrm{Li}$ et al. 2009). We demonstrated that the genetic and physical interactions we observed between Sgdlp and Fallp correlate with their collaborative function in $18 \mathrm{~S}$ rRNA biogenesis. First, we showed that the CS yeast strain $f a 11(\mathrm{~T} / \mathrm{V})$ displays pre-rRNA processing defects at sites $\mathrm{A} 0, \mathrm{~A} 1$, and $\mathrm{A} 2$, resulting in a decrease in $18 \mathrm{~S}$ [the $18 \mathrm{~S} / 25 \mathrm{~S}$ ratio in the $f a 11(\mathrm{~T} / \mathrm{V})$ strain is $75 \%$ of that in the wild-type strain] (Fig. 6A, top panel) and 20S (Fig. 6A, bottom panel) rRNA levels, as well as accumulation of $23 \mathrm{~S}$ rRNA (Fig. 6A, middle and bottom panels) and $35 \mathrm{~S}$ rRNA primary transcript (Fig. 6A, middle and bottom panels). Second, we observed that the suppressor mutation $s g d 1$ (S340Y) in part rescues the rRNA biogenesis defects of $f a 11(\mathrm{~T} / \mathrm{V})$ : It partially restores mature $18 \mathrm{~S}$ rRNA [the $18 \mathrm{~S} / 25 \mathrm{~S}$ ratio in the suppressor strain fal1(T/V) $\operatorname{sgd} 1(\mathrm{~S} / \mathrm{Y})$ is $85 \%$ of that in the wild-type strain] (Fig. 6A, top panel) and 20S (Fig. 6A, bottom panel) pre-rRNA levels, and partially alleviates the increases in $35 \mathrm{~S}$ primary transcript (Fig. 6A, middle and bottom panels) and $23 \mathrm{~S}$ rRNA (Fig. 6A, middle and bottom panels). Third, consistent with Fallp and Sgdlp acting together in 18S rRNA biogenesis, depletion of Sgd1p results in pre-rRNA processing defects identical to those of Fallp: Slow growth (Supplemental Fig. S7B,C) of a yeast strain (Supplemental Fig. S7A) expressing Sgd1p from the GAL1 promoter in medium containing glucose was accompanied by drastic decreases in $18 \mathrm{~S}$ (Fig. 6B, top panel; Supplemental Fig. S7D) and 20S (Fig. 6B, bottom panel) rRNA levels, as well as accumulation of $23 \mathrm{~S}$ rRNA (Fig. 6B, middle and bottom panels) and $35 \mathrm{~S}$ rRNA primary transcript (Fig. 6B, middle and bottom panels).

Furthermore, consistent with evolutionary conservation of the function of the Sgd1p/Fallp complex from yeast to humans, siRNA-mediated knockdown of eIF4AIII or NOM1 (Supplemental Fig. S11) resulted in a decreased rate of $18 \mathrm{~S}$ rRNA formation in HEK293T cells. When we in vivo pulse-labeled cells with ${ }^{3} \mathrm{H}$-methyl-methionine followed by a chase with cold methionine, we observed lower amounts of $18 \mathrm{~S}$ rRNA (Fig. 7B,C, cf. lanes 1-5 and 7-11). Also decreased was $41 \mathrm{~S}$ pre-rRNA at 15 and $30 \mathrm{~min}$ of chase (Fig. 7B,C, cf. the amounts of $41 \mathrm{~S}$ pre-rRNA in lanes 2,3 and 8,9) and 21S pre-rRNA (Fig. 7B,C, small bottom panels, cf. the amounts of $21 \mathrm{~S}$ pre-rRNA in lanes $1-5$ and 
7-11); formation of both 41S and 21S pre rRNA (Fig. 7A) requires cleavage at site 1 , which creates the $5^{\prime}$ end of the mature 18S rRNA. A decrease in the ratio of newly synthesized ( ${ }^{3} \mathrm{H}$-labeled) mature small-subunit (18S) rRNA to the large-subunit (28S) rRNA (as observed after $3 \mathrm{~h}$ of chase) can be seen in NOM1 knockdown cells (Fig. 7C, cf. the $18 \mathrm{~S} / 28 \mathrm{~S}$ ratio in lanes 6 and 12). A processing delay at site 2 is observed in the eIF4AIII knockdown cells, as judged by an increased ratio of unprocessed $45 \mathrm{~S}$ pre-rRNA to $30 \mathrm{~S} / 32 \mathrm{~S}$ pre-rRNA during the first hour of chase (Fig. 7B, cf. the ratio in lanes 4,5 and 10,11). To confirm specificity of the siRNAs used for knockdowns, we cotransfected knockdown cells with
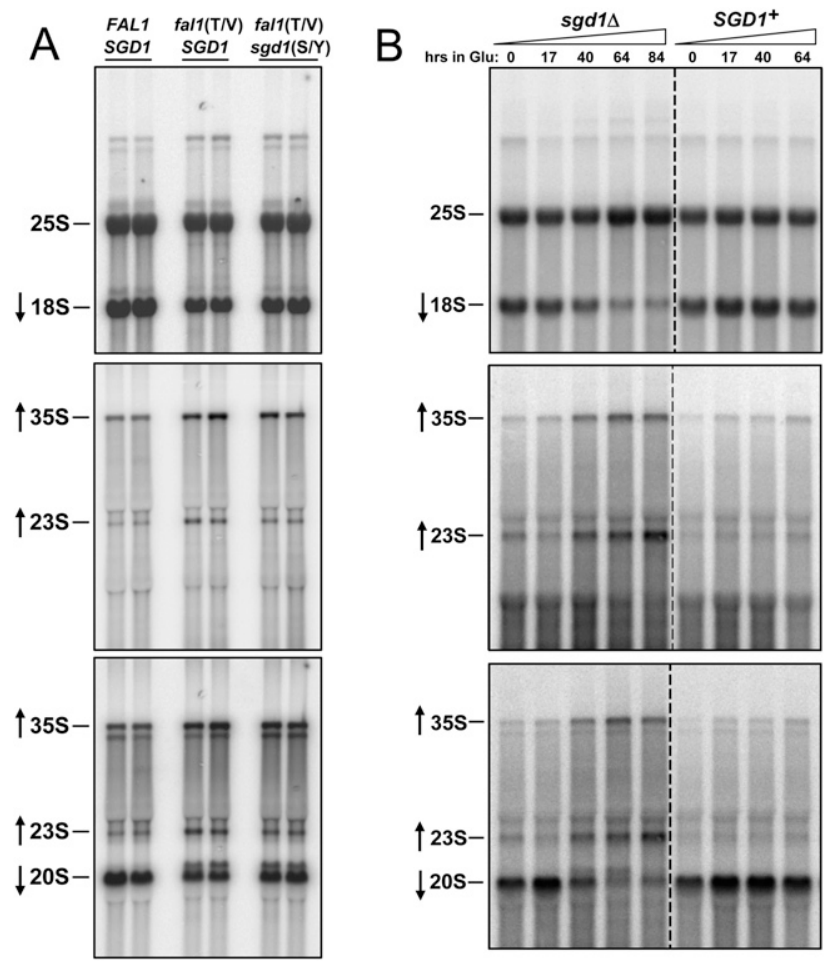

Figure 6. The extragenic suppressor mutation sgd1(S340Y) alleviates 18S rRNA biogenesis defects of the CS fal1(T322V) strain; $S G D 1$ is required for rRNA processing at the same sites (A0, A1, and A2) as FAL1. (A) The 18S rRNA biogenesis defect of the $S$. cerevisiae CS strain fal1(T322V) and its suppression by sgd1(S340Y) are visualized by Northern blot using DNA oligonucleotide probes L2 and L9 (top panel), L1 (middle panel), and L3 (bottom panel); probes are shown in Supplemental Figure S2. Total RNA was prepared from strains grown as in Figure 3A. (B) $S G D 1$ is required for $18 \mathrm{~S}$ rRNA biogenesis at sites $\mathrm{A} 0, \mathrm{~A} 1$, and A2 (same sites as Fallp). Depletion of $\mathrm{P}_{G A L 1}$-expressed Sgd1p in glucose-containing media at $16^{\circ} \mathrm{C}$ results in a decrease in $18 \mathrm{~S}$ rRNA levels (top panel), an increase in 23S rRNA levels (middle panel), a decrease in $20 \mathrm{~S}$ rRNA levels (bottom panel), and accumulation of the $35 \mathrm{~S}$ rRNA primary transcript (middle and bottom panels) in the yeast sgd1s strain (Northern blot was probed as in $A$; schematics of the strains are shown in Supplemental Fig. S7A, and growth curves are shown in Supplemental Fig. S7B). Arrows on the left indicate changes in the levels of rRNAs or intermediates in $f a 11(\mathrm{~T} / \mathrm{V})(A)$ and $\operatorname{sgd} 1 \Delta(B)$ strains compared with wild type.
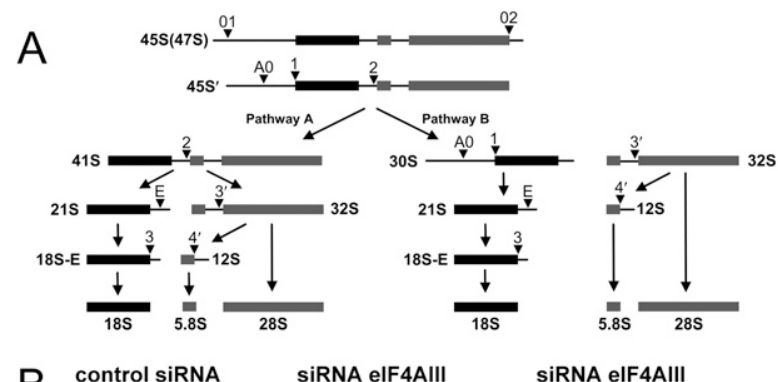

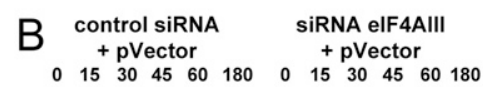
t pelF4AIII

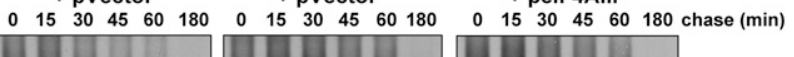
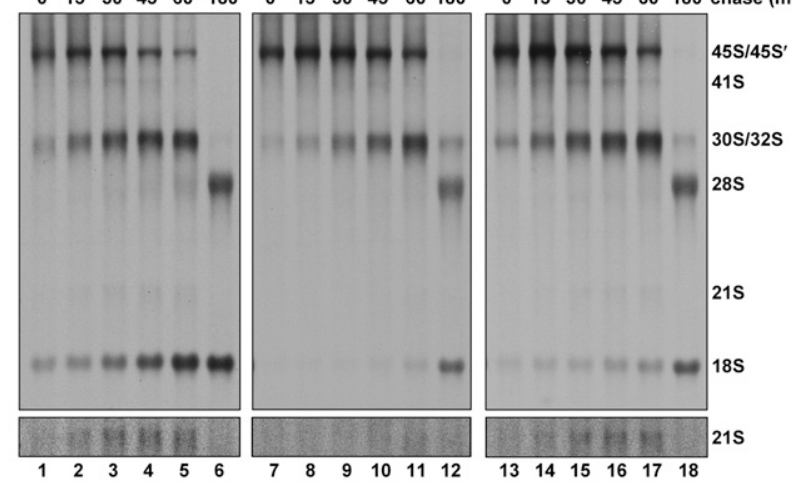

control siRnA
+ pVector
0
$15 \quad 30 \quad 45 \quad 60 \quad 180$

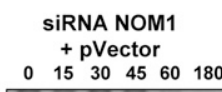

SIRNA NOM1 + pNOM1r

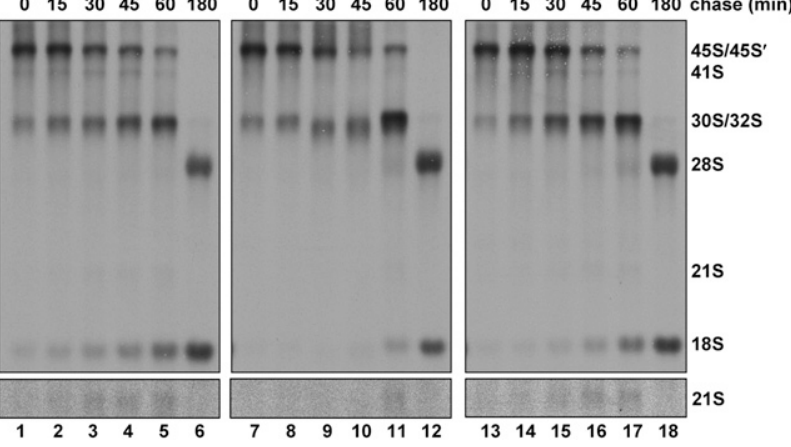

Figure 7. siRNA-mediated knockdown of either eIF4AIII or NOM1 results in an 18S rRNA biogenesis defect in human cells. (A) Schematic of rRNA processing in humans. (B) siRNAmediated knockdown of eIF4AIII results in a decreased rate of $18 \mathrm{~S}$ rRNA formation, as determined by in vivo pulse-labeling of HEK293T cells with [methyl- $\left.{ }^{3} \mathrm{H}\right]$-methionine followed by chase with cold methionine. (Middle panel) Processing of $45 \mathrm{~S}$ and formation of $41 \mathrm{~S}, 30 \mathrm{~S} / 32 \mathrm{~S}$, and $21 \mathrm{~S}$ pre-RNAs are delayed, resulting in a decrease in mature $18 \mathrm{~S}$ rRNA, suggesting defects at sites 1 and 2. (Right panel) Cotransfection of a plasmid expressing an siRNA-resistant version of eIF4AIII partially rescues the rate of formation of $18 \mathrm{~S}$ rRNA and precursors, confirming specificity. $(C)$ siRNA-mediated knockdown of NOM1 results in a decreased rate of $18 \mathrm{~S}$ rRNA formation, as determined by pulsechase performed as in B. (Middle panel) Formation of 41S and 21S pre-RNAs is delayed, resulting in a decrease in mature 18S rRNA, suggesting a processing defect at site 1 . Consistently, a decreased ratio of mature small (18S) to large (28S) subunit rRNA is observed after $3 \mathrm{~h}$ of chase (cf. lanes 6 and 12). (Right panel) Cotransfection of a plasmid expressing an siRNA-resistant variant of NOM1 partially rescues the rate of appearance of $18 \mathrm{~S}$ rRNA as well as $21 \mathrm{~S}$ and $41 \mathrm{~S}$ precursors. 
plasmids expressing siRNA-resistant eIF4AIII (peIF4AIII ${ }^{\mathrm{r}}$ ) (Fig. 7B, right panel) and NOM1 (pNOM1 ${ }^{\mathrm{r}}$ ) (Fig. $7 \mathrm{C}$, right panel), which resulted in alleviation of each of the observed processing defects, or an empty vector (Fig. 7B,C, middle panels).

\section{Discussion}

We identified the eIF4G-like human protein NOM1 as a conserved interacting partner of the eIF4A-like protein eIF4AIII. This interaction in the yeast $S$. cerevisiae (Fallp/ Sgd1p) and humans (eIF4AII/NOM1) is similar to the established eIF4A/eIF4G interaction within eIF4F. In yeast, each of the two partner proteins is essential and is required for $18 \mathrm{~S}$ rRNA biogenesis. In addition to their physical interaction and involvement in the same steps of $18 \mathrm{~S}$ rRNA biogenesis, we established that Fallp and Sgdlp are genetically and functionally tied via a dominant compensatory suppressor relationship: Whereas mutation T322V in an RNA-interacting residue of Fallp results in cold sensitivity and a defect in yeast 18S rRNA biogenesis, the dominant allele-specific extragenic suppressor mutation S340Y in Sgd1p alleviates these defects. The suppressor mutation in the large protein Sgd1p (899 amino acids) spontaneously appeared in the MIF4G domain (the major eIF4A-interacting domain of eIF4G) within a 12-amino-acid conserved motif found in eIF4G and NOM1 orthologous proteins that represents "the largest contiguous interaction surface" between eIF4G and eIF4A (Schutz et al. 2008). The two identified $S G D 1$ mutations that interact genetically with FAL1 (extragenic suppressor mutation S340Y and synthetic mutation $\mathrm{N} 343 \mathrm{Y}$ ) replace conserved Sgd1p amino acids that were previously shown for yeast eIF4G (S612 and N615) to each form two direct intermolecular hydrogen bonds with eIF4A, based on the X-ray structure of the yeast eIF4A/eIF4G complex (Schutz et al. 2008).

Our results illuminate a novel function as well as the evolutionary origin of the eIF4AIII protein. Previously reported functions of eIF4AIII in the EJC, NMD, neuronal protein expression, and selenocysteine regulation (Giorgi et al. 2007; Isken and Maquat 2008; Budiman et al. 2009) were not sufficient to explain the high degree of conservation of the eIF4AIII family $160 \%$ identity between human eIF4AIII and yeast Fallp over the entire protein length) and Walker A and B boxes within these proteins. This was because (1) no EJC has been identified in yeast (Tange et al. 2004), (2) the function of eIF4AIII in NMD is not conserved in D. melanogaster and C. elegans (Palacios et al. 2004; Longman et al. 2007), and (3) other components of the EJC are conserved only in metazoans (Tange et al. 2004).

We uncovered the conserved ability of human and $D$. melanogaster eIF4AIII to function in 18S rRNA biogenesis upon heterologous expression of these proteins in an $S$. cerevisiae strain lacking the essential yeast DEAD-box helicase Fallp. Restoration of both strain viability and 18S rRNA biogenesis was specific, since even the most closely related human eIF4AI and eIF4AII and yeast Tif1 proteins did not complement fal1s. We further showed that the complementation of the fal1s phenotype by human eIF4AIII requires intact Walker A and B boxes, identifying the first function of eIF4AIII that strictly depends on ATP hydrolysis (at least when eIF4AIII is expressed heterologously in S. cerevisiae). The other known functions of eIF4AIII-EJC formation, NMD activation, and binding to the GPx1 SECIS element-are not abolished by such mutations despite the loss of ATPase and consequent helicase activity in vitro (Shibuya et al. 2006; Zhang and Krainer 2007; Budiman et al. 2009). Finally, we showed conservation of the function of the Fallp/Sgd1p complex in 18S rRNA biogenesis from yeast to humans by demonstrating pre-rRNA processing defects arising from siRNA-mediated knockdown of eIF4AIII and NOM1 in HEK293T cells. Interestingly, even though knockdown of either eIF4AIII or NOM1 similarly resulted in decreased rates of formation of the mature 18S rRNA and a similar processing defect at site 1 (formation of $41 \mathrm{~S}$ and $21 \mathrm{~S})$, delayed processing of $45 \mathrm{~S}$ pre-rRNA at site 2 (which is specific for eIF4AIII knockdown) may reflect a specific requirement for eIF4AIII but not NOM1 at site 2 . Alternatively, another factor may substitute for NOM1 in processing at site 2 (but not at site 1) following NOM1 down-regulation, or, possibly, the degree of NOM1 knockdown was not sufficient to observe the defect in site 2 processing.

The versatility displayed by eIF4AIII in interacting with two different sets of partners is only beginning to be appreciated as a feature of DEAD-box helicases. Mammalian eIF4AIII interacts on the one hand with Magoh and Y14, as the core protein that deposits the EJC on newly spliced mRNAs in the nucleoplasm, and on the other hand with NOM1 in the nucleolus. Whether these distinct associations are dictated simply by its dual subcellular localization is not yet clear. Interestingly, eIF4AIII has been reported to reside in nucleoli in plants (Pendle et al. 2005), and some human eIF4AIII mutants surprisingly localize to the nucleolus rather than the nucleoplasm (Shibuya et al. 2006). Other DEAD-box helicases that have been implicated in more than one cellular process are Prp43 in splicing and rRNA processing (Lebaron et al. 2005), eIF4A in translation initiation and regulation of SMAD degradation in D. melanogaster (Li and Li 2006), and Dbp5 in mRNA export as well as translation termination (Gross et al. 2007). The lack of a requirement for ATP hydrolysis in the release of eIF4AIII (the EJC) from its mRNA substrates can perhaps be ascribed to the power of the ribosome progressing along the mRNA during translation; in rRNA biogenesis, ATP hydrolysis presumably leads to the release of eIF4AIII from its rRNA substrate, allowing progression to the next step in the processing pathway. It will be interesting to learn whether other RNA helicases also have differential requirements for ATP hydrolysis in performing their multiple cellular roles.

Our results are consistent with the idea that eIF4G and NOM1 evolved from a common ancestor protein (Marintchev and Wagner 2005). Whereas eIF4G functions with eIF4AI/II in translation initiation in eukaryotes, the human eIF4G-like protein NOM1 evolved to cooperate with a very similar protein, eIF4AIII, but in a completely 
different process: $18 \mathrm{~S}$ rRNA biogenesis. It is possible that additional partners for other DEAD-box helicases that exhibit interactions similar to the eIF4AIII/NOM1 and Fallp/Sgdlp pairs will be identified in the future.

In retrospect, our initial lack of success in identifying Sgdlp as a Fallp-interacting protein using TAP purification of Fallp is not surprising. Instead, it highlights the similarity of the Fallp/Sgdlp interaction to the relatively weak, but functionally crucial, interaction of eIF4A with eIF4G within eIF4F. Specifically, eIF4A can be readily dissociated from mammalian eIF4G on ion exchange columns (Ray et al. 1985; Etchison and Milburn 1987) and can exchange with free eIF4A (Yoder-Hill et al. 1993). Binding of eIF4A to eIF4G is weaker than that of eIF4E to eIF4G (Gingras et al. 1999). Purified eIF4F complexes from plant (Lax et al. 1985), D. melanogaster (Sierra and Zapata 1994), and yeast (Goyer et al. 1989) can lack eIF4A, presumably because eIF4A dissociates during isolation. Thus, we switched to identifying other Sgdlp/Fallpassociated components using in vivo formaldehyde crosslinking followed by affinity selection using TAP-tagged Sgd1p (in yeast) and Flag-tagged NOM1 (without crosslinking, in human cells). Mass-spectrometric analysis of proteins copurifying with NOM1-Flag from human cell nuclear extract identified 24 proteins, 18 of which are known ribosomal proteins (five small and 13 large subunits) (Supplemental Fig. S6B). The presence of many $\mathrm{r}$ proteins in NOM1 preparations may indicate an association of NOM1 with preribosomes at stages preceding the cleavage of the $35 \mathrm{~S}$ pre-rRNA transcript (consistent with the reported nucleolar localization of NOM1 [Simmons et al. 2005] and its yeast ortholog, Sgd1p [Akhtar et al. 2000]), or with both the small and large ribosomal subunits at later nuclear steps of rRNA processing. Remarkably, endogenous human eIF4AIII was one of the 24 proteins identified by mass spectrometry as copurifying with NOM1Flag (Fig. 5C; Supplemental Fig. S6B). We are currently investigating the exact composition of the eIF4AII/NOM1 complex.

The apparent difference in the interaction strength of human NOM1 with eIF4AIII relative to yeast Sgdlp with Fall is similar to that of eIF4G with eIF4A in these two organisms: Human eIF4G and eIF4A interact much more strongly than their yeast counterparts (Gingras et al. 1999). This difference cannot be explained by the lack of the second C-terminal eIF4A-binding region (the MA3 domain) in yeast eIF4G, since both yeast Sgd1p and human NOM1 have similar structures; each possesses one MIF4G and one MA3 domain (Fig. 4A; Ponting 2000; Marintchev and Wagner 2005). Further biochemical studies may explain why detection of the Sgdlp/Fallp interaction in yeast requires formaldehyde cross-linking, whereas robust copurification of human eIF4AIII with NOM1-Flag from nuclear extracts of human cells occurs without cross-linking.

The CS defect of the fal1(T322V) strain and its extragenic suppression by $\operatorname{sgd1}(\mathrm{S} 340 \mathrm{Y})$, as well as the synthetic growth defect of the fal1(T322V) sgd1(N343Y) double mutant, require further investigation. The mutated threonine residue T322 of Fallp is conserved in numerous
DEAD-box helicases beyond the eIF4A family (Tanner and Linder 2001). In crystal structures of both the EJC core (Andersen et al. 2006; Bono et al. 2006) and VASA complexed with RNA (Sengoku et al. 2006), this threonine forms hydrogen bonds with RNA backbone phosphates. The T546A mutation in VASA /corresponding to T322 in Fallp) abolishes the in vitro ATPase and helicase activities of VASA, and results in a $50 \%$ decrease in crosslinking efficiency to the $U_{10}$ oligomer (Sengoku et al. 2006). The eIF4A/eIF4G interaction model suggests that the suppressing (S340) and synthetically interacting (N343) residues of Sgdlp contact Fallp on the side opposite the putative RNA-binding domain (Fig. 4B). One possibility is that each of these mutations in Sgdlp (S340Y or N343Y) acts through a direct interaction with Fallp at the Sgdlp/ Fallp interface to induce a slight conformational change in Fallp(T322V) that increases function at a lower temperature. Mechanistically, suppression through a conformational change in the RNA-binding region of Fallp induced by Sgdlp binding to the opposite side of Fallp would be consistent with the model (Oberer et al. 2005) of eIF4G serving as a soft clamp to stabilize the relative orientation of the two helicase domains and favoring the closed "active" conformation of eIF4A. Such a mechanism would also be consistent with numerous reports of the stimulation of eIF4A helicase activity upon eIF4G binding (Marintchev and Wagner 2004). Alternatively, it is possible that the genetically interacting residues of Sgd1p (S340Y or N343Y) make direct contact with the CS residue of Fallp (T322), although a gross structural rearrangement would be required. Detailed biochemical characterization in the context of other members of the Sgdlp/Fallp complex may shed light on the mechanism of fal1(T322V) cold sensitivity and extragenic suppression.

Human NOM1 was originally identified as a nucleolar eIF4A-binding protein encoded within the chromosome $7 \mathrm{q} 36$ breakpoint region found in the majority of pediatric acute myeloid leukemia (AML) patients carrying the $\mathrm{t}(7 ; 12)$ translocation (Simmons et al. 2005; von Bergh et al. 2006), which is associated with particularly poor prognosis. The genomic segment (7q35-7q36) has also been implicated as a critical region in del(7q) leukemias (Dohner et al. 1998). Despite the proximity $(<1 \mathrm{~kb})$ of the NOM1 gene to the translocation breakpoint $t(7 ; 12)(\mathrm{q} 36 ; \mathrm{p} 13)$ (Simmons et al. 2005), NOM1 mRNA levels are not affected (von Bergh et al. 2006). Whether NOM1's proximity to the breakpoint contributes to AML will require further study.

\section{Materials and methods}

Strains and plasmids

Strains and plasmids are described in the Supplemental Material.

Yeast growth

Yeast strains shown in Figure 2B were grown in synthetic medium lacking uracil and containing $2 \%$ galactose and $2 \%$ rafinose (SGR-ura) and shifted to synthetic medium lacking uracil and containing $2 \%$ dextrose and $2 \%$ rafinose (SDR-ura). 
Strains in Figure 2C and Supplemental Figure S7, B and C, were grown in SGR-his and SGR-ura media, respectively, and then shifted to SGR-his/SDR-his and SGR-ura/SDR-ura media, respectively.

\section{Construction and isolation of TS and CS mutants}

Mutations in Fallp at specific positions (149, 152, 304, and 322) were generated using PCR and QuickChange-based methods using DNA oligonucleotides with specific or degenerate (NNN) triplets. Mutations in Fallp at random positions were generated using error-prone PCR using Taq polymerase and elevated $\mathrm{Mg}^{2+}$. Libraries of resulting fal1*-HIS5 cassettes (Supplemental Fig. S9B) were linearized and transformed into yeast, the FAL1(WT)containing plasmid was counterselected, and the growth of the resulting strains was assessed at different temperatures. The phenotypes of isolated mutants [ fal1 (CS), fal1 $\left(\mathrm{CS}_{2}\right)$, fal1(TS), and fal1 $\left(\mathrm{TS}_{2}\right)$ ] were confirmed by tetrad analysis (Supplemental Figs. S3D, S9E) and complementation with FAL1(WT) (Supplemental Figs. S3E, S9C).

\section{Northern blot}

RNA separated on formaldehyde agarose $(1 \%)$ was transferred to Zeta-Probe membrane (Bio-Rad), cross-linked using $\mathrm{UV}_{254}$ (Stratalinker 2400), and visualized with synthetic $5^{\prime}$-end ${ }^{32} \mathrm{P}$ labeled DNA oligonucleotide probes (Supplemental Fig. S2).

\section{Protein purification}

Protein purification is described in the Supplemental Material.

\section{Western blot}

Western Lightning Plus-ECL Enhanced Chemiluminescence Substrate from PerkinElmer and SuperSignal West Pico/Femto Maximum Sensitivity Substrates from Pierce were used for detection.

\section{Mass spectrometry}

Flag-eluted preparations of NOM1 were separated on $4 \%-20 \%$ SDS PAGE and stained with SYPRO Ruby, and gel slices were analyzed by ESI-LC-MS/MS at Columbia University Protein Core Facility.

\section{Software used}

Sequences were aligned using Multalin (Corpet 1988), and were shaded with Boxshade 3.21.

\section{Acknowledgments}

We thank Shobha Vasudevan, Nikolay Kolev, Mei-Di Shu, Sumit Borah, and Abiodun Kukoyi for help, valuable discussions, and sharing reagents. We also thank Adrian Krainer for sharing antibody against human eIF4AIII, Christine Guthrie for critically reading the manuscript, Angie Miccinello for editorial help, and the rest of the Steitz laboratory for stimulating discussions. This work was supported by grant GM026154 from the NIH. J.A.S is an investigator of the Howard Hughes Medical Institute.

\section{Note added in proof}

While this manuscript was in review, the laboroatory of Ulrike Kutay reported a high-throughput identification of 153 human proteins whose siRNA depletion affected rRNA biogenesis in HeLa cells; human eIF4AIII was listed as one of these proteins (Wild et al. 2010).

\section{References}

Akhtar N, Pahlman AK, Larsson K, Corbett AH, Adler L. 2000. SGD1 encodes an essential nuclear protein of Saccharomyces cerevisiae that affects expression of the GPD1 gene for glycerol 3-phosphate dehydrogenase. FEBS Lett 483: 87-92.

Andersen CB, Ballut L, Johansen JS, Chamieh H, Nielsen KH, Oliveira CL, Pedersen JS, Seraphin B, Le Hir H, Andersen GR. 2006. Structure of the exon junction core complex with a trapped DEAD-box ATPase bound to RNA. Science 313: 1968-1972.

Ballut L, Marchadier B, Baguet A, Tomasetto C, Seraphin B, Le Hir H. 2005. The exon junction core complex is locked onto RNA by inhibition of eIF4AIII ATPase activity. Nat Struct Mol Biol 12: 861-869.

Bleichert F, Baserga SJ. 2007. The long unwinding road of RNA helicases. Mol Cell 27: 339-352.

Bono F, Ebert J, Lorentzen E, Conti E. 2006. The crystal structure of the exon junction complex reveals how it maintains a stable grip on mRNA. Cell 126: 713-725.

Budiman ME, Bubenik JL, Miniard AC, Middleton LM, Gerber CA, Cash A, Driscoll DM. 2009. Eukaryotic initiation factor $4 \mathrm{a} 3$ is a selenium-regulated RNA-binding protein that selectively inhibits selenocysteine incorporation. Mol Cell 35: 479-489.

Corpet F. 1988. Multiple sequence alignment with hierarchical clustering. Nucleic Acids Res 16: 10881-10890.

Dohner K, Brown J, Hehmann U, Hetzel C, Stewart J, Lowther G, Scholl C, Frohling S, Cuneo A, Tsui LC, et al. 1998. Molecular cytogenetic characterization of a critical region in bands 7q35-q36 commonly deleted in malignant myeloid disorders. Blood 92: 4031-4035.

Etchison D, Milburn S. 1987. Separation of protein synthesis initiation factor eIF4A from a p220-associated cap binding complex activity. Mol Cell Biochem 76: 15-25.

Fairman ME, Maroney PA, Wang W, Bowers HA, Gollnick P, Nilsen TW, Jankowsky E. 2004. Protein displacement by DExH/D 'RNA helicases' without duplex unwinding. Science 304: 730-734.

Gatfield D, Unterholzner L, Ciccarelli FD, Bork P, Izaurralde E. 2003. Nonsense-mediated mRNA decay in Drosophila: at the intersection of the yeast and mammalian pathways. EMBO I 22: 3960-3970.

Gingras AC, Raught B, Sonenberg N. 1999. eIF4 initiation factors: effectors of mRNA recruitment to ribosomes and regulators of translation. Annu Rev Biochem 68: 913-963.

Giorgi C, Yeo GW, Stone ME, Katz DB, Burge C, Turrigiano G, Moore MJ. 2007. The EJC factor eIF4AIII modulates synaptic strength and neuronal protein expression. Cell 130: 179191.

Gonzalez CI, Bhattacharya A, Wang W, Peltz SW. 2001. Nonsense-mediated mRNA decay in Saccharomyces cerevisiae. Gene 274: 15-25.

Goyer C, Altmann M, Trachsel H, Sonenberg N. 1989. Identification and characterization of cap-binding proteins from yeast. J Biol Chem 264: 7603-7610.

Gross T, Siepmann A, Sturm D, Windgassen M, Scarcelli JJ, Seedorf M, Cole CN, Krebber H. 2007. The DEAD-box RNA helicase Dbp5 functions in translation termination. Science 315: 646-649.

Hinnebusch AG. 2006. eIF3: a versatile scaffold for translation initiation complexes. Trends Biochem Sci 31: 553-562. 
Isken O, Maquat LE. 2008. The multiple lives of NMD factors: balancing roles in gene and genome regulation. Nat Rev Genet 9: 699-712.

Kressler D, de la Cruz J, Rojo M, Linder P. 1997. Fallp is an essential DEAD-box protein involved in 40S-ribosomal-subunit biogenesis in Saccharomyces cerevisiae. Mol Cell Biol 17: 7283-7294.

Lax S, Fritz W, Browning K, Ravel J. 1985. Isolation and characterization of factors from wheat germ that exhibit eukaryotic initiation factor 4B activity and overcome 7-methylguanosine 5 '-triphosphate inhibition of polypeptide synthesis. Proc Natl Acad Sci 82: 330-333.

Lebaron S, Froment C, Fromont-Racine M, Rain JC, Monsarrat B, Caizergues-Ferrer M, Henry Y. 2005. The splicing ATPase prp43p is a component of multiple preribosomal particles. Mol Cell Biol 25: 9269-9282.

Le Hir H, Izaurralde E, Maquat LE, Moore MJ. 2000a. The spliceosome deposits multiple proteins 20-24 nucleotides upstream of mRNA exon-exon junctions. EMBO J 19: 68606869.

Le Hir H, Moore MJ, Maquat LE. 2000b. Pre-mRNA splicing alters mRNP composition: evidence for stable association of proteins at exon-exon junctions. Genes Dev 14: 1098-1108.

Le Hir H, Gatfield D, Izaurralde E, Moore MJ. 2001. The exonexon junction complex provides a binding platform for factors involved in mRNA export and nonsense-mediated mRNA decay. EMBO J 20: 4987-4997.

Li J, Li WX. 2006. A novel function of Drosophila eIF4A as a negative regulator of Dpp/BMP signalling that mediates SMAD degradation. Nat Cell Biol 8: 1407-1414.

Li Q, Imataka H, Morino S, Rogers GW Jr, Richter-Cook NJ, Merrick WC, Sonenberg N. 1999. Eukaryotic translation initiation factor 4AIII (eIF4AIII) is functionally distinct from eIF4AI and eIF4AII. Mol Cell Biol 19: 7336-7346.

Li Z, Lee I, Moradi E, Hung NJ, Johnson AW, Marcotte EM. 2009. Rational extension of the ribosome biogenesis pathway using network-guided genetics. PLOS Biol 7: e1000213. doi: 10.1371/journal.pbio.1000213.

Linder P. 2003. Yeast RNA helicases of the DEAD-box family involved in translation initiation. Biol Cell 95: 157-167.

Longman D, Plasterk RH, Johnstone IL, Caceres JF. 2007. Mechanistic insights and identification of two novel factors in the C. elegans NMD pathway. Genes Dev 21: 1075-1085.

Lykke-Andersen J, Shu MD, Steitz JA. 2001. Communication of the position of exon-exon junctions to the mRNA surveillance machinery by the protein RNPS1. Science 293: 18361839.

Maeda I, Kohara Y, Yamamoto M, Sugimoto A. 2001. Large-scale analysis of gene function in Caenorhabditis elegans by highthroughput RNAi. Curr Biol 11: 171-176.

Marintchev A, Wagner G. 2004. Translation initiation: structures, mechanisms and evolution. Q Rev Biophys 37: 197284.

Marintchev A, Wagner G. 2005. eIF4G and CBP80 share a common origin and similar domain organization: implications for the structure and function of eIF4G. Biochemistry 44: 12265-12272.

Morino S, Imataka H, Svitkin YV, Pestova TV, Sonenberg N. 2000. Eukaryotic translation initiation factor 4E (eIF4E) binding site and the middle one-third of eIF4GI constitute the core domain for cap-dependent translation, and the C-terminal one-third functions as a modulatory region. Mol Cell Biol 20: 468-477.

Nott A, Le Hir H, Moore MJ. 2004. Splicing enhances translation in mammalian cells: an additional function of the exon junction complex. Genes Dev 18: 210-222.
Oberer M, Marintchev A, Wagner G. 2005. Structural basis for the enhancement of eIF4A helicase activity by eIF4G. Genes Dev 19: 2212-2223.

Palacios IM, Gatfield D, St Johnston D, Izaurralde E. 2004. An eIF4AIII-containing complex required for mRNA localization and nonsense-mediated mRNA decay. Nature 427: 753 757.

Pendle AF, Clark GP, Boon R, Lewandowska D, Lam YW, Andersen J, Mann M, Lamond AI, Brown JW, Shaw PJ. 2005. Proteomic analysis of the Arabidopsis nucleolus suggests novel nucleolar functions. Mol Biol Cell 16: 260-269.

Ponting CP. 2000. Novel eIF4G domain homologues linking mRNA translation with nonsense-mediated mRNA decay. Trends Biochem Sci 25: 423-426.

Puig O, Caspary F, Rigaut G, Rutz B, Bouveret E, BragadoNilsson E, Wilm M, Seraphin B. 2001. The tandem affinity purification (TAP) method: a general procedure of protein complex purification. Methods 24: 218-229.

Ray BK, Lawson TG, Kramer JC, Cladaras MH, Grifo JA, Abramson RD, Merrick WC, Thach RE. 1985. ATP-dependent unwinding of messenger RNA structure by eukaryotic initiation factors. I Biol Chem 260: 7651-7658.

Rigaut G, Shevchenko A, Rutz B, Wilm M, Mann M, Séraphin B. 1999. A generic protein purification method for protein complex characterization and proteome exploration. Nat Biotechnol 17: 1030-1032.

Rocak S, Linder P. 2004. DEAD-box proteins: the driving forces behind RNA metabolism. Nat Rev Mol Cell Biol 5: 232241.

Rozen F, Edery I, Meerovitch K, Dever TE, Merrick WC, Sonenberg N. 1990. Bidirectional RNA helicase activity of eucaryotic translation initiation factors 4A and 4F. Mol Cell Biol 10: 1134-1144.

Schutz P, Bumann M, Oberholzer AE, Bieniossek C, Trachsel H, Altmann M, Baumann U. 2008. Crystal structure of the yeast eIF4A-eIF4G complex: an RNA-helicase controlled by protein-protein interactions. Proc Natl Acad Sci 105: 9564-9569.

Sengoku T, Nureki O, Nakamura A, Kobayashi S, Yokoyama S. 2006. Structural basis for RNA unwinding by the DEAD-box protein Drosophila Vasa. Cell 125: 287-300.

Shibuya T, Tange TO, Stroupe ME, Moore MJ. 2006. Mutational analysis of human eIF4AIII identifies regions necessary for exon junction complex formation and nonsense-mediated mRNA decay. RNA 12: 360-374.

Sierra JM, Zapata JM. 1994. Translational regulation of the heat shock response. Mol Biol Rep 19: 211-220.

Simmons HM, Ruis BL, Kapoor M, Hudacek AW, Conklin KF. 2005. Identification of NOM1, a nucleolar, eIF4A binding protein encoded within the chromosome $7 \mathrm{q} 36$ breakpoint region targeted in cases of pediatric acute myeloid leukemia. Gene 347: 137-145.

Solem A, Zingler N, Pyle AM. 2006. A DEAD protein that activates intron self-splicing without unwinding RNA. Mol Cell 24: 611-617.

Tange TO, Nott A, Moore MJ. 2004. The ever-increasing complexities of the exon junction complex. Curr Opin Cell Biol 16: 279-284.

Tanner NK, Linder P. 2001. DExD/H box RNA helicases: from generic motors to specific dissociation functions. Mol Cell 8: 251-262.

Tanner NK, Cordin O, Banroques J, Doere M, Linder P. 2003. The Q motif: a newly identified motif in DEAD box helicases may regulate ATP binding and hydrolysis. Mol Cell 11: 127-138.

von Bergh AR, van Drunen E, van Wering ER, van Zutven LJ, Hainmann I, Lonnerholm G, Meijerink JP, Pieters R, Beverloo 
Alexandrov et al.

HB. 2006. High incidence of $\mathrm{t}(7 ; 12)(\mathrm{q} 36 ; \mathrm{p} 13)$ in infant AML but not in infant ALL, with a dismal outcome and ectopic expression of HLXB9. Genes Chromosomes Cancer 45: 731-739.

Wild T, Horvath P, Wyler E, Widmann B, Badertscher L, Zemp I, Kozak K, Csucs G, Lund E, Kutay U. 2010. A protein inventory of human ribosome biogenesis reveals an essential function of exportin 5 in 60S subunit export. PLOS Biol 8: e1000522. doi: 10.1371/journal.pbio.1000522.

Yang Q, Jankowsky E. 2005. ATP- and ADP-dependent modulation of RNA unwinding and strand annealing activities by the DEAD-box protein DED1. Biochemistry 44: 1359113601.

Yoder-Hill J, Pause A, Sonenberg N, Merrick WC. 1993. The p46 subunit of eukaryotic initiation factor (eIF)-4F exchanges with eIF-4A. J Biol Chem 268: 5566-5573.

Zhang Z, Krainer AR. 2007. Splicing remodels messenger ribonucleoprotein architecture via eIF4A3-dependent and -independent recruitment of exon junction complex components. Proc Natl Acad Sci 104: 11574-11579. 


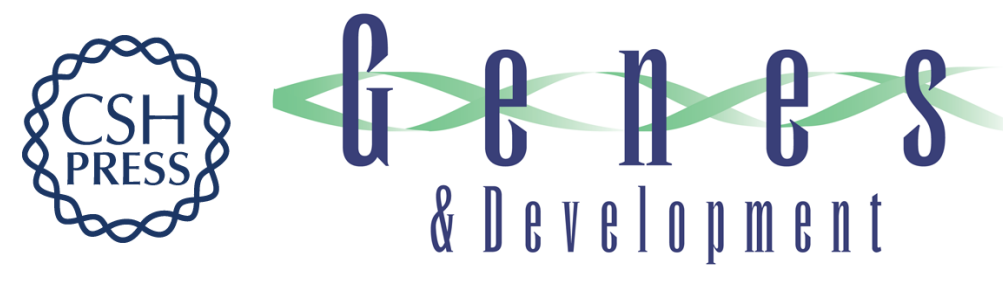

\section{Human elF4AIII interacts with an elF4G-like partner, NOM1, revealing an evolutionarily conserved function outside the exon junction complex}

Andrei Alexandrov, David Colognori and Joan A. Steitz

Genes Dev. 2011, 25:

Access the most recent version at doi:10.1101/gad.2045411

Supplemental http://genesdev.cshlp.org/content/suppl/2011/05/10/25.10.1078.DC1

Material

References This article cites 58 articles, 25 of which can be accessed free at:

http://genesdev.cshlp.org/content/25/10/1078.full.html\#ref-list-1

License

Email Alerting Receive free email alerts when new articles cite this article - sign up in the box at the top

Service right corner of the article or click here.

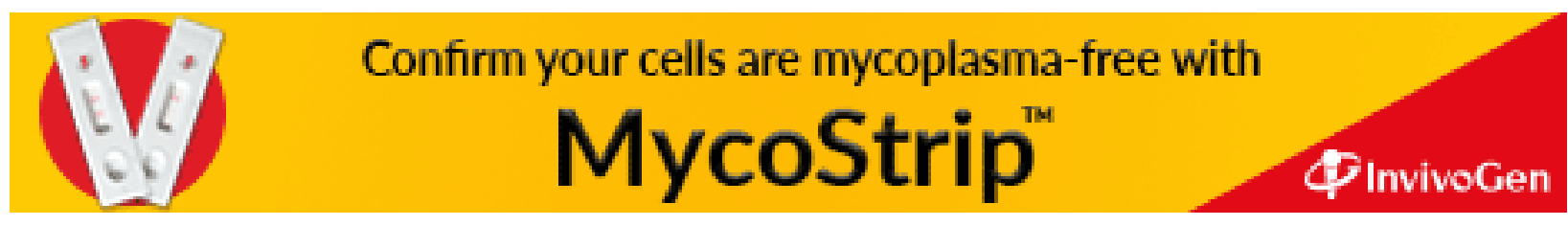

